\title{
Relative changes in blood flow with functional electrical stimulation during exercise of the paralyzed lower limbs
}

\author{
W Phillips ${ }^{1}$, LN Burkett ${ }^{2}, \mathrm{R}_{\text {Munro }}{ }^{2}, \mathrm{M} \mathrm{Davis}^{2}$ and K Pomeroy ${ }^{3}$ \\ ${ }^{1}$ Stanford University Medical School; ${ }^{2}$ Arizona State University; ${ }^{3}$ Phoenix Physical Medicine Center, USA
}

Eight spinal cord injured (SCI) patients performed three sets of exercise with two conditions, $60 \%$ and $80 \%$ of $\mathrm{VO}_{2 \text { peak }}$, with an arm crank ergometer. Functional neuromuscular stimulation was used to induce static leg contractions in two of the above sets of exercise. The three exercise sets were performed with no functional neuromuscular stimulation (NOS); with functional neuromuscular stimulation at 40 milliamperes; and with functional neuromuscular stimulation at 80 milliamperes (HIS). The lower limb blood flow was estimated by a photoelectric plethysmograph. Results showed that the lower limb blood flow was consistently reduced across both functional neuromuscular stimulation levels (17.4\% from NOS to LOS; $13.8 \%$ from LOS to HIS; and $28.8 \%$ from NOS to HIS), and work loads $\left(15.3 \%\right.$ from rest to $60 \% \mathrm{VO}_{2 \text { peak }} ; 38.0 \%$ from $60 \% \mathrm{VO}_{2 \text { peak }}$ to $80 \%$ $\mathrm{VO}_{2 \text { peak }}$; and $47.5 \%$ from rest to $\left.80 \% \mathrm{VO}_{2 \text { peak }}\right)$. Rate-pressure product was decreased by $8.3 \%$ between NOS and HIS at $60 \% \mathrm{VO}_{2 \text { peak }}(15.7 \pm 3.4$ to $14.4 \pm 3.8)$, by $6.8 \%$ between NOS and HIS at $80 \% \mathrm{VO}_{2 \text { peak }}(18.9 \pm 53$ to $17.6 \pm 4.8)$, and by $12.4 \%$ between LOS and HIS at $80 \% \mathrm{VO}_{2 \text { peak }}(20.1 \pm 6.7$ to $17.6 \pm 4.8)$. These data indicate that in SCI (a) functional neuromuscular stimulation-induced contractions of the lower limb muscles can increase blood flow and thus reduce venous blood pooling in the paralyzed muscles, and (b) such improvements are associated with a reduced rate pressure product. In practical terms, this represents a potential enhancement of cardiovascular training responses, and thus health status, over that gained from conventional arm exercises. It has also been suggested that functional neuromuscular stimulation may provide a more 'natural' means of ameliorating blood pooling, since the active contraction of the paralyzed muscles may also enhance their integrity.

Keywords: functional electrical stimulation; exercise; paralyzed lower limbs; blood flow

\section{Introduction}

Currently, many countries in the world have embraced the importance of exercise and its relationship to health. This awareness of the importance of exercise includes those individuals who have a spinal cord injury (SCI). Participation in fitness activities of those with a SCI has risen each year with the myriad of activities now available for them and with this trend has come an increased demand for relevant research and for physiological data. Much of this increased research has focused on the impaired lower limb blood flow (causing blood pooling) found in such people. This pooling caused by an absent or diminished sympathetic tone of blood vessels and the flaccid lower limb musculature, when combined with the effects of gravity, results in relatively high levels of passive vasodilation. ${ }^{1}$ Such a condition is likely to have a considerable effect on the adaptation to exercise of SCI individuals.

The first appearance of testing physiological adaptations to physical stress for individuals with a SCI was

Correspondence: Lee N Burkett, Department ESPE, Arizona State University, Box 870701, Tempe, Arizona 85287-0701, USA recorded following World War II. Wakim et $a l^{2}$ studied the upper body blood flow change of able bodied (AB) and disabled bodied (DB) subjects following weight training. Burkett et $\mathrm{al}^{3}$ studied the blood flow in exercising wheelchair bound individuals. They found that about half of the SCI subjects had increased blood flow in the legs during arm exercise. In recent years, the use of functional neuromuscular stimulation (FNS) to attenuate impaired venous return in SCI individuals has been suggested. Rattan et al $^{4}$ demonstrated that isometric stimulation of both calf and thigh musculature increasingly activated the venous 'muscle pump'. FNS has been associated with indirect measurement of blood flow (no instrument was used to measure blood flow) in those with a SCI, both at rest and during exercise. ${ }^{5-9}$ Only Burkett et $a^{3}$ measured blood flow with a photoelectric plethysmograph; all other studies deduced an increase in blood flow (and thus a possible reduction in venous pooling) by an increase in central hemodynamic responses, such as stroke volume (SV) and cardiac output (Q). To date instrumentation designed to estimate blood flow and the effect of FNS on blood flow in the lower limbs has not been used in research studies. The purpose of the current study was 
to use a photoelectric plethysmograph to measure the effect of FNS-induced isometric contractions on lower limb blood flow during arm exercise in SCI patients.

\section{Methods}

Eight healthy subjects with SCI volunteered for this study (age 33.1 \pm 7.5 , range $21-45$ years; lesion levels from C6 to T12). Seven of the subjects were classified as motor complete and two had a degree of sensation in the lower limbs. All subjects responded to FNS with a visible muscular contraction. All subjects signed an informed consent form (in accordance with the Arizona State University Human Subjects Institutional Review Board), and were cleared by their physician for participation in the experiment. Prior to testing the subjects were asked to desist from eating for $4 \mathrm{~h}$. They were also requested to avoid vigorous exercise for $24 \mathrm{~h}$ before the testing sessions. All subjects underwent an initial period of familiarization with the arm crank ergometer (ACE) and FNS equipment.

After familiarization all subjects performed a maximal ACE test with no electrical stimulation. Following the maximal ACE test the subjects returned on 3 separate days and performed three submaximal exercise sets (with two conditions, $60 \%$ and $80 \%$ of $\mathrm{VO}_{2 \text { peak }}$ ) with an ACE. The three submaximal exercise sets were performed as follows; (a) with no FNS, (NOS), (b) with FNS at 40 milliamperes (LOS), and (c) with FNS at 80 milliamperes (HIS). It should be noted that a FNS of 40 milliamperes could elicit a small visible static contraction, while the FNS of 80 milliamperes could elicit a large visible static contraction. The three stimulation conditions were randomly applied across the exercise sessions. The submaximal $(60 \%$ and $80 \%$ $\mathrm{VO}_{2 \text { peak }}$ ) exercises consisted of: (a) a 2 min pre-exercise rest period, (b) a warm up for $3 \mathrm{~min}$ at a gradually increasing work load (WL), (c) 5 min at the 'target' WL $\left[60 \% \mathrm{VO}_{2 \text { peak }}\right.$ or $\left.80 \% \mathrm{VO}_{2 \text { peak }}\right]$, and (d) a graduated cool down of $3 \mathrm{~min}$. There was a $10 \mathrm{~min}$ rest period between the $60 \%$ and $80 \% \mathrm{VO}_{2 \text { peak }}$ submaximal tests.

An audible metronomic beeper was used to maintain arm crank speed at 50 RPM. The ACE used in the study was a Combi System 5 Cardiac Rehabilitation Cycle (Combi Co. Ltd, 16-9 Uchikanda 3-Chome, Chiyoda-Ku, Tokyo, 101, Japan), which had been modified for arm cranking. Metabolic indices were monitored continuously by open circuit spirometry using a Horizon metabolic cart (Sensormedica Corporation, Yorba Linda, California 92687-9923, USA).

Electrical stimulation was applied via bifurcated leads and self adhesive reusable surface electrodes. The electrodes were applied over the motor point (both legs) on the following muscles; (a) quadriceps, (b) hamstrings, (c) gastrocnemius, and (d) tibialis. Two EMPI 'Focus' neuromuscular stimulators ${ }^{10}$ (EMPI Inc. St Paul, Minnesota 55112, USA) were used to induce the electrical stimulation. Electrical stimulation was allied with symmetrical biphasic pulses of $300 \mu \mathrm{s}$ at $35 \mathrm{~Hz}$ delivered across a $1000 \mathrm{ohm}$ load, over a 'duty cycle' of $2.5 \mathrm{~s}$ 'on' and $5 \mathrm{~s}$ 'off', with a $2 \mathrm{~s}$ 'rise' and 'fall' time for each pulse. These settings were consistent with the recommendations from the Rancho Amigos Rehabilitation book on functional electrical stimulation and EMPI. ${ }^{10,11}$

Indices of lower limb peripheral blood flow were monitored with a photoelectric plethysmograph (vascular analyzer Model 2100. Vascular Diagnostic Instruments, Inc, Cherry Hill, New Jersey 08003, USA). The sensing probe of the plethysmograph (PEP), which was attached to the left big toe of each subject, contains a tiny light source and a photosensitive cell that responds to the light reflected from arterial blood in the peripheral vascular bed over which the sensor is placed. Since the cell responds linearly to the light reflected from the blood in its view, the amount of pulsating light it registers is proportional to the volume of pulsating arterial blood flow. Due to the nature of its design, the blood flows recorded by the PEP, show relative, as opposed to quantitative changes. All data were recorded on a graphical strip at $25 \mathrm{~mm} \mathrm{~min}^{-1}$. The amplitude setting on the plethysmograph remained constant throughout each session. Blood flow was determined from the graphical data by estimating the area (mean of five pulse traces in $\mathrm{mm}^{2}$ ) under the anacrotic wave. Recordings of blood flow were taken during the ACE exercise session as follows: (a) at rest; (b) immediately post exercise at $60 \%$ and $80 \%$ of $\mathrm{VO}_{2 \text { peak }}$; (c) $60 \%$ and $80 \% \quad \mathrm{VO}_{2 \text { peak }}$ cool down; (d) 10 min following the $60 \%$ and $80 \% \mathrm{VO}_{2 \text { peak }}$ cool down. Overall differences between the conditions were analyzed with a repeated measures MANOVA. Significant main effects were followed by a single factor ANOVA, and differences between factor levels were determined with Tukey's HSD post hoc tests (Table 1). Results for the $\mathrm{VO}_{2}$ were reported in another publication. ${ }^{12}$

\section{Results}

Post exercise peripheral blood flow (Figure 1) was consistently reduced across FNS levels. Mean value decreases of $17.4 \%, 13.8 \%$ and $28.8 \%$ were found between NOS and LOS, effect size $(E S)=0.4$. LOS and HIS $(\mathrm{ES}=0.2)$, and NOS and HIS $(\mathrm{ES}=0.4)$, respectively. Decreases in peripheral blood flow were also noted across work loads. Mean value decreases of $15.3 \%, 38.0 \%$ and $47.5 \%$ were found between rest and $60 \% \mathrm{VO}_{2 \text { peak }},(\mathrm{ES}=0.2), 60 \% \mathrm{VO}_{2 \text { peak }}$ and $80 \% \quad \mathrm{VO}_{2 \text { peak }},(\mathrm{ES}=0.5)$ and rest and $80 \% \mathrm{VO}_{2 \text { peak }}$ $(\mathrm{ES}=0.7)$, respectively. Rate-pressure product (RPP) decreased by $8.3 \%$ between NOS and HIS at $60 \%$ $\mathrm{VO}_{2 \text { peak }}(15.7 \pm 3.4$ to $14.4 \pm 3.8)$, by $6.8 \%$ between NOS and HIS at $80 \% \mathrm{VO}_{2 \text { peak }}(18.9 \pm 53$ to $17.6 \pm 4.8)$ and by $12.4 \%$ between LOS and HIS at $80 \% \mathrm{VO}_{2 \text { peak }}$ $(20.1 \pm 6.7$ to $17.6 \pm 4.8)$. ES of $0.4,0.3$ and 0.4 , respectively, were calculated for these three differences.

\section{Discussion}

Venous pooling in SCI individuals has been established as a contributing factor to a lack of aerobic 
Table 1 Multifactor repeated measures MANOVA for blood flow

\begin{tabular}{|c|c|c|c|c|c|c|}
\hline Source & $S S$ & $d f$ & $M S$ & $F$ & $P$ & Sign \\
\hline Blocks/subjects & 2009.40 & 6 & 334.90 & 1.57 & 0.1717 & No \\
\hline Exercise & 9099.37 & 2 & 4549.69 & 21.55 & 0.0000 & Yes \\
\hline Stimulation & 3755.48 & 2 & 1877.74 & 8.90 & 0.0005 & Yes \\
\hline Exercise stimulation & 2528.03 & 4 & 632.01 & 2.99 & 0.0277 & Yes \\
\hline Error & 10135.76 & 48 & 211.16 & & & \\
\hline Total & 2752.03 & 62 & & & & \\
\hline
\end{tabular}

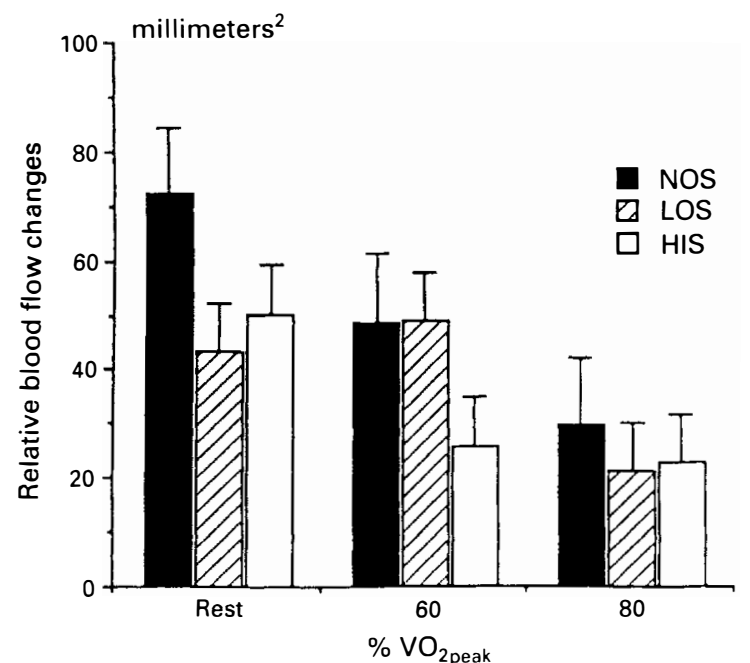

Figure 1 Blood flow responses to ACE versus ACE + FNS at two different exercise intensities (mean \pm SEM)

response, ${ }^{12-14}$ as has its consequent deleterious effects on central and peripheral circulation..$^{1,14}$ Studies examining the effects of FNS on venous return have generally used central hemodynamic responses as indications of blood pooling. Figoni et $\mathrm{al}^{7}$ demonstrated a significant increase in $\mathrm{Q}$ and $\mathrm{SV}$ at various levels of passive tilt during ACE + FNS compared to ACE alone, and Davis et $a l^{5}$ in a similar study also showed improvements in these same parameters. The increase in these measures was considered to be secondary to a decrease in blood pooling which, in turn, was secondary to the external stimulus of FNS. In contrast to these measures, the plethysmograph used in this study and by the Burkett $e t$ al $^{3}$ study gives a much better indication of blood flow, and thus the possible blood pooling in the lower limbs than has been shown in previous studies, since it is sensitive to relative changes in the light reflected from blood passing through a peripheral capillary bed, and thus to relative changes in peripheral vasodilation. Although large decreases in mean values of peripheral blood flow measures were obtained across all levels of FNS and WL in the current study, there was also a large variability found in the data. The large variability was probably due to occasional involuntary, and seemingly random leg spasms, which were seen to occur during some test sessions. These movements may have interfered with the plethysmographic response traces for the affected subjects, and thus with the consistency of their blood flow calculation. The trend found in this study (Figure 1) for a reduction in peripheral blood flow, coincident with the application of FNS, lends support to previous research findings using other measures. ${ }^{5-7}$ Further support is provided by the ES calculated for blood flow increase between NOS and LOS (0.4) and between NOS and HIS (0.4). Support for the increased blood flow (and thus less blood pooling) is also given by moderate correlations found between blood flow values and $\mathrm{VO}_{2}$ uptake at $60 \% \mathrm{VO}_{2 \text { peak }}$ with HIS $(r=-0.5)$, and at $80 \% \mathrm{VO}_{2 \text { peak }}$ with both LOS $(r=-0.5)$ and HIS $(r=-0.4)$.

The reduction in peripheral blood pooling, caused by increased blood flow, via FNS application, has been likened to a mechanical, 'milking', effect on the lower limb venous bed, ${ }^{8}$ this theory being supported by the similar results obtained by other authors using a variety of external compression techniques such as elastic stockings, ${ }^{13}$ pressure boots,,$^{14}$ and, anti-G suits. ${ }^{13}$

A beneficial effect of FNS found in this study, which has not been reported by other research workers, was an improved RPP $(8.3 \%$ decrease between NOS and HIS at $60 \% \mathrm{VO}_{2 \text { peak }} ; 6.8 \%$ and $12.4 \%$ decrease at $80 \%$ $\mathrm{VO}_{2 \text { peak }}$ between NOS and HIS and between LOS and HIS respectively). A reduced RPP would indicate a more efficient myocardial $\mathrm{O}_{2}$ uptake with FNS, and thus improved efficiency of the cardiorespiratory system.

\section{Clinical implications}

Although the effects of FNS-induced lower limb static contractions on cardiovascular parameters has received comparatively little attention, considerable benefits to those with a SCI could potentially be available from this mode of exercise. Previous studies have suggested that the improvement of central hemodynamic responses, secondary to a reduction of blood pooling via FNS, may make more blood available to the upper body musculature, and thus provide a platform for increased physical fitness training., ${ }^{1,12,14}$ The results of this study support these findings and additionally suggest that such increases in blood availability may increase the efficiency of myocardial $\mathrm{O}^{2}$ uptake. In practical terms, this represents a potential enhancement of cardiovascular training responses, and thus health status, over that gained from conventional arm exercise. It has also been suggested that FNS may 
provide a more 'natural' means of increasing blood flow, thus ameliorating blood pooling, since the active contraction of the paralyzed muscles may also enhance their integrity. ${ }^{1,14}$

In addition to the metabolic aspects above, there are a number of reinforcing factors for the SCI person to utilize the ACE + FNS mode of exercise. No subject incurred any problems with the skin or was unable to complete the exercise because the electrical stimulation was uncomfortable. The small commercial neuromuscular stimulators and self adhesive, reusable electrodes, used in this study are relatively inexpensive and easy to use. The use of these small and inexpensive units allow $\mathrm{ACE}+\mathrm{FNS}$ to be performed in the performer's own wheelchair. The setting on the FNS used in this study $^{10,11}$ closely resembles natural muscular contraction. It is suggested that individuals who wish to use FNS units use the same settings used in this study. ACE equipment is becoming more available in health clubs and gymnasiums, and an increasing range of inexpensive portable units are being marketed for home use. These data indicate that ACE + FNS should be seriously considered as part of a health and fitness program for wheelchair bound individuals.

\section{Acknowledgement}

This study was supported by EMPI Inc.

\section{References}

1 Glaser RM et al. Use of lower-limb FNS to improve arm exercise performance of SCI individuals. Proceedings of the 16th Annual Conference of RESNA, 1993.
2 Wakim KG, Elkins E, Worden R, Polley H. The effects of therapeutic exercise on peripheral circulation of normal and paraplegic individuals. Arch Phys Med Rehabil 1949; 30: 86-95.

3 Burkett LN et al. Blood flow and lactic acid levels in exercising paralyzed wheelchair bound individuals. Adapt Phys Educ $Q$ 1988; 5: 60-73.

4 Rattan SW, Glaser RM, Collins SR. Hemodynamic effects of electrically stimulated leg muscles (abstract). Fed Proc 1985; 44: 1379.

5 Davis GM et al. Cardiovascular responses to FNS-induced isometric leg exercise during orthostatic stress in paraplegics. Proceedings of the International Conference of the Association for the Advancement of Rehabilitation Technology, 1988; Montreal, Canada, pp 326-327.

6 Figoni SF, Glaser RM, Rodgers MM. Hemodynamic responses of quadriplegics to arm, ES-leg, and combined arm and ES-leg ergometry. Med Sci Sports Exerc 1989; 21 (Suppl): S96.

7 Figoni SF et al. FNS-assisted venous return in exercising SCI men. Proceedings of the International Conference of the Association of Advanced Rehabilitation Technology, 1988; 3: 328-329.

8 Glaser RM et al. Central hemodynamic responses to lower-limb FNS. Proceedings of the IEEE/Ninth Annual Conference of the Engineering in Medicine and Biology Society, 1987; pp 0615-0617.

9 Poninchtera-Mulcare JA et al. Effects of leg FNS on wheelchair locomotion effort. Proceedings of the 16th Annual Conference of RESNA, 1993.

10 EMPI Incorporated (1989). Clinical Guidelines. EMPI Inc: St Paul, Minnesota.

11 Benton LA, Baker LL, Bowman BR, Waters RL. Functional Electrical Stimulation-A Practical Clinical Glide. Rancho Los Amigos Rehabilitation Engineering Center: Downey, California.

12 Phillips W, Burkett LN. Arm crank exercise with static leg FNS in spinal cord injured persons. Med Sci Sports Exerc (in press).

13 Pitetti KH, Barrett PJ, Cambell KD. The effects of an anti-G suit on the exercise responses of spinal cord injured individuals. Med Sci Sports Exerc 1992; 24 (Suppl): S18.

14 Thomas AJ, Davis GM, Gass GC. Cardiorespiratory responses to FES-induced leg muscle contractions during arm exercise in paraplegics. Med Sci Sports Exerc 1992; 24 (Suppl): S18. 